\title{
PERCEPCIONES DE LOS CIRUJANOS MÁXILOFACIALES CHILENOS ACERCA DEL DOBLE GRADO MÉDICO-DENTAL*
}

\author{
Drs. Ricardo Cartes-Velásquez ${ }^{1,2}$, Hernán Ramírez S. ${ }^{3}$, Juan Cortés A.,5 \\ 1 Programa de Doctorado en Ciencias Médicas, Universidad de la Frontera, Temuco. \\ 2 Facultad de Odontología, Universidad de Concepción, Concepción. \\ 3 Departamento de Cirugía Oncológica y Máxilofacial, División de Cirugía, Facultad de Medicina, Pontificia \\ Universidad Católica de Chile, Santiago. \\ ${ }^{4}$ Facultad de Odontología, Universidad de Chile, Santiago. \\ 5 Hospital San Borja Arriarán, Santiago. \\ Chile.
}

\begin{abstract}
Perceptions of chilean oral and maxillofacial surgeons about dual-degree

Introduction: Dual-degree (DD) in oral and maxillofacial surgery still remains a controversial and/ or unsolved issue in many countries, although there is a growing trend towards its implementation in the developed world. The aim of this research is to describe the perceptions of Chilean oral and maxillofacial surgeons about DD and their position regarding the need of its implementation in Chile. Methodology: A cross-sectional survey was performed via the LimeSurvey web platform, included all surgeons registered in the Chilean Society. The questionnaire comprised two sections dedicated to surgical practice and perceptions about DD, respectively. Results: 67\% (61) of surgeons completed the survey, main proportion men with more than 5 years of surgical practice, and experience in training teaching. $55.74 \%$ (34) considered the implementation of DD programs in Chile necessary. There are correlations between this pro-DD position and hospital practice, knowledge about $\mathrm{DD}$, the need for more medical skills/knowledge in daily practice, the need for DD to perform the full scope of OMS, and the opinion that Chilean training programs are not practicing at an International level. Conclusion: In Chile, as in other countries, there is a dichotomic view of DD, and it appears absolutely possible to initiate a propositive discussion about it.
\end{abstract}

Key words: "Education, Medical [MeSH]", "Education, Dental [MeSH], dual-degree, maxillofacial.

\section{Resumen}

Introducción: El doble grado médico-dental (DG) en cirugía máxilofacial continúa siendo una cuestión controversial y sin resolver en muchos países, aunque hay una fuerte tendencia hacia su implementación en el mundo desarrollado. El objetivo de este trabajo es describir la percepción de los cirujanos máxilofaciales chilenos acerca del DG y su postura frente a la necesidad de implementarlo en el país. Metodología: En-

*Recibido el 2 de mayo de 2013 y aceptado para publicación el 12 de agosto de 2013.

Conflictos de interés: Ninguno.

Correspondencia: Dr. Ricardo Cartes-Velásquez

Los Olivos 37, Penco. CP 4140265.

ricardo@cartesvelasquez.com 
cuesta electrónica, realizada en la plataforma web LimeSurvey, incluyendo todos los cirujanos registrados en la Sociedad de Cirugía y Traumatología Bucal y Máxilofacial de Chile. El cuestionario incluyó 2 secciones: práctica quirúrgica y percepciones del DG. Resultados: 67\% (61) cirujanos completaron la encuesta, la mayoría hombres, con más de 5 años de práctica quirúrgica y experiencia docente en la especialidad. 55,74\% (34) consideran que la implementación de programas DG es necesaria en Chile. Hay asociación de la postura pro-DG y la práctica hospitalaria, conocimiento del doble grado, necesidad de mayores habilidades/conocimiento médico en la práctica diaria, necesidad del DG para practicar el campo completo de la especialidad y la opinión que los programas de especialización chilenos no están al nivel internacional. Conclusión: En Chile, como en otros países, hay una visión dicotómica del DG y parece perfectamente posible iniciar una discusión propositiva del tema.

Palabras clave: Educación médica, educación dental, doble grado, máxilofacial.

\section{Introducción}

La doble formación médico-dental en cirugía máxilofacial (CMF), aquella en que es necesaria la titulación como médico-cirujano y cirujano-dentista para obtener la especialidad, comúnmente conocida como doble grado $(\mathrm{DG})^{1}$, continúa siendo un tema controversial y no resuelto en muchos países, aunque en los últimos años se ha dado una fuerte tendencia hacia su implementación en el mundo desarrollado, especialmente en Europa ${ }^{2}$ y en una menor proporción en Norteamérica ${ }^{3}$.

La evidencia sugiere que el DG es ventajoso para la práctica de la CMF por los siguientes aspectos: campo clínico extendido (cirugía oncológica, reconstructiva y microcirugía), acceso a subespecialidades (fellowships) y sociedades científicoprofesionales, generación de nuevo conocimiento y evidencia (publicaciones en revistas de corriente principal), prerrogativas quirúrgicas, respaldo legal y reconocimiento social; aunque existen aspectos negativos, como la extensión y el costo de una larga formación profesional que puede durar hasta 18 años (incluyendo medicina, odontología y especialización), lo que parece injustificado e inalcanzable para la realidad de países del tercer mundo ${ }^{1}$.

Actualmente, en Latinoamérica no existen programas de especialización de DG y ninguno de los chilenos, basados únicamente en el título odontológico, cumple a cabalidad con los requerimientos planteados en la guía de especialización en CMF de la Asociación Internacional de Cirujanos Orales y Máxilofaciales ${ }^{4}$.

El objetivo de este trabajo es describir la percepción de los cirujanos máxilofaciales chilenos acerca del DG y su postura frente a la necesidad de implementarlo en el país.

\section{Metodología}

\section{Diseño y muestra}

Estudio de corte transversal, se realizó una encuesta electrónica durante abril y mayo de 2012.
Se incluyeron todos los cirujanos registrados en la Sociedad de Cirugía y Traumatología Bucal y Máxilofacial de Chile (SCTBM) con una dirección de correo electrónica válida. No se consideraron criterios de exclusión.

\section{Recolección}

La encuesta fue implementada en la plataforma web LimeSurvey. Se envío una invitación inicial a todos los cirujanos, además de 5 recordatorios cada 10 días para aquellos que no habían respondido, con el objetivo de alcanzar la mayor tasa de respuesta.

\section{Variables y análisis}

La encuesta comprendía 2 secciones: la primera relativa a datos personales y práctica quirúrgica, mientras que la segunda se enfocó a las percepciones acerca del DG (Tabla 1). Todas las preguntas eran de tipo cerrado, con excepción de la última, en que se preguntaba en términos generales cual era la percepción de los cirujanos acerca de los programas de especialización de DG. Se realizó estadística descriptiva, incluyendo frecuencias y porcentajes, para el grupo general y de acuerdo a su posición, positiva o negativa, de la necesidad de implementar un programa de DG en Chile.

\section{Aspectos éticos}

La investigación contó con la revisión y autorización del Directorio de la SCTBM. La encuesta incluía en su portada un consentimiento informado, recalcando el carácter anónimo y confidencial de los datos entregados, se consideró como aceptación de dicho consentimiento la respuesta del instrumento.

\section{Resultados}

Noventa y uno de los 105 correos electrónicos registrados por la SCTBM fueron válidos, de los cuales un $67 \%$ (61) completó la encuesta. La mayoría correspondió a hombres $(91,80 \%)$, con más de 5 años de experiencia quirúrgica en CMF $(81,97 \%)$ y 
Tabla 1. Preguntas y respuestas de la encuesta para la muestra general y de acuerdo a su posición frente a la implementación del doble grado en Chile

\begin{tabular}{|c|c|c|c|}
\hline & \multicolumn{3}{|c|}{$\begin{array}{l}\text { ¿Cree necesario contar con programas de formación } \\
\text { de doble grado en cirugía máxilofacial en Chile? }\end{array}$} \\
\hline & Sí & No & General \\
\hline $\begin{array}{l}\text { Su práctica es mayoritari } \\
\text { Cirugía bucal } \\
\text { Cirugía máxilofacial }\end{array}$ & $\begin{array}{c}0 \%(0) \\
57,63 \%(34)\end{array}$ & $\begin{array}{c}100 \%(2) \\
42,37 \%(25)\end{array}$ & $\begin{array}{c}3,28 \%(2) \\
96,72 \%(59)\end{array}$ \\
\hline $\begin{array}{l}\text { Sexo: } \\
\text { Femenino } \\
\text { Masculino }\end{array}$ & $\begin{array}{c}0 \%(0) \\
60,71 \%(34)\end{array}$ & $\begin{array}{c}100 \%(5) \\
39,29 \%(22)\end{array}$ & $\begin{array}{c}8,2 \%(5) \\
91,8 \%(56)\end{array}$ \\
\hline $\begin{array}{l}\text { Años de práctica quirúrg } \\
\text { Menos de } 5 \\
5 \text { a } 15 \\
\text { Más de } 15\end{array}$ & $\begin{array}{c}54,55 \%(6) \\
56 \%(14) \\
56 \%(14)\end{array}$ & $\begin{array}{c}45,45 \%(5) \\
44 \%(11) \\
44 \%(11)\end{array}$ & $\begin{array}{l}18,03 \%(11) \\
40,98 \%(25) \\
40,98 \%(25)\end{array}$ \\
\hline $\begin{array}{l}\text { Lugar de ejercicio princi } \\
\text { Hospital } \\
\text { Ambulatorio }\end{array}$ & $\begin{array}{l}59,09 \%(26) \\
47,06 \%(8)\end{array}$ & $\begin{array}{l}40,91 \%(18) \\
52,94 \%(9)\end{array}$ & $\begin{array}{l}72,13 \%(44) \\
27,87 \%(17)\end{array}$ \\
\hline $\begin{array}{l}\text { Su dependencia principa } \\
\text { Pública } \\
\text { Privada }\end{array}$ & $\begin{array}{l}54,84 \%(17) \\
56,67 \%(17)\end{array}$ & $\begin{array}{l}45,16 \%(14) \\
43,33 \%(13)\end{array}$ & $\begin{array}{l}50,82 \%(31) \\
49,18 \%(30)\end{array}$ \\
\hline $\begin{array}{l}\text { ¿Ha tenido experiencia d } \\
\text { Sí } \\
\text { No }\end{array}$ & $\begin{array}{l}\text { ión de cirujanos máxi } \\
52,17 \%(24) \\
66,67 \%(10)\end{array}$ & $\begin{array}{l}47,83 \%(22) \\
33,33 \%(5)\end{array}$ & $\begin{array}{l}75,41 \%(46) \\
24,59 \%(15)\end{array}$ \\
\hline $\begin{array}{l}\text { ¿Conocía la doble forma } \\
\text { Sí } \\
\text { No }\end{array}$ & $\begin{array}{c}\text { en cirugía máxilofaci } \\
57,14 \%(32) \\
40 \%(2)\end{array}$ & $\begin{array}{l}42,86 \%(24) \\
\quad 60 \%(3)\end{array}$ & $\begin{array}{l}91,8 \%(56) \\
8,2(5)\end{array}$ \\
\hline $\begin{array}{l}\text { ¿Conoce algún programa } \\
\text { Sí } \\
\text { No }\end{array}$ & $\begin{array}{l}\text { en el extranjero? } \\
63,04 \%(29) \\
33,33 \%(5)\end{array}$ & $\begin{array}{l}36,96 \%(17) \\
66,67 \%(10)\end{array}$ & $\begin{array}{l}75,41 \%(46) \\
24,59 \%(15)\end{array}$ \\
\hline $\begin{array}{l}\text { ¿Considera necesaria la } \\
\text { Sí } \\
\text { No }\end{array}$ & $\begin{array}{l}\text { a ejercer el campo co } \\
87,1 \%(27) \\
23,33 \%(7)\end{array}$ & $\begin{array}{c}\text { pecialidad? }^{*} \\
12,9 \%(4) \\
76,67(23)\end{array}$ & $\begin{array}{l}50,82 \%(31) \\
49,18 \%(30)\end{array}$ \\
\hline $\begin{array}{l}\text { En su práctica diaria ¿Ha } \\
\quad \text { Sí } \\
\text { No }\end{array}$ & $\begin{array}{c}\text { ontar con mayores cor } \\
75,68 \%(28) \\
25 \%(6)\end{array}$ & $\begin{array}{c}\text { bilidades médi } \\
\text { 24,32\%(9) } \\
75 \%(18)\end{array}$ & $\begin{array}{c}60,66 \%(37) \\
39,34(24)\end{array}$ \\
\hline $\begin{array}{l}\text { En su opinión ¿Los prog } \\
\text { Sí } \\
\text { No }\end{array}$ & $\begin{array}{r}\text { nacionales preparan } \\
39,39 \%(13) \\
75 \%(21)\end{array}$ & $\begin{array}{c}\text { aivel internacic } \\
60,61 \%(20) \\
25 \%(7)\end{array}$ & $\begin{array}{l}54,1 \%(33) \\
45,9 \%(28)\end{array}$ \\
\hline
\end{tabular}

*De acuerdo a las guías de la IAOMS. http://www.iaoms.org/TrainingGuidelines.aspx?display=Training.

con experiencia docente en la formación de especialistas en CMF $(75,41 \%)$.

Un 55,74\% (34) de los cirujanos encuestados consideran que la implementación de un programa de DG en CMF es necesaria en Chile. Los resultados para las respuestas cerradas se muestran en la Tabla 1.

Las respuestas a la última pregunta abierta tuvieron una fuerte relación con las preguntas previas, mostrando una postura favorable o crítica acerca del DG y su posible implementación en Chile. Respuestas positivas incluyeron expresiones recurrentes como: "Preparación total del especialista", "Permiten formación sólida en el ámbito quirúrgico y médico, lo que le da al cirujano las competencias para actuar con propiedad, independencia y respaldo legal", "Son buenos ya que cumplen con todas las normas internacionales" y "Es necesario para mejorar las competencias". Desde la postura negativa las expresiones comunes fueron: "Demasiado 
largo e injustificado", "Le quita sustento a los CMF dentistas, dando la impresión a los médicos que no estamos bien formados", "Sobre calificación profesional, que no condice con la práctica profesional que requiere el país" y "Son muy extensos y no se justifican. La formación médico odontológica ya se ha probado y no da buenos resultados".

\section{Discusión}

En la última Conferencia Internacional en CMF (20 th International Conference on Oral and Maxillofacial Surgery), realizada en Chile (1-4 de noviembre de 2011), la formación de los futuros especialistas en el siglo $\mathrm{XXI}^{5}$ y en Sudamérica ${ }^{6}$ fueron temas de discusión, incluyendo tópicos como qué tan lejos ha avanzado la especialidad y hacia dónde debe ir, concluyendo que los cirujanos máxilofaciales necesitan un proceso de formación más integral y estandarizado.

Sin embargo, la estructura y ruta del proceso formativo, así como el rol del DG, permanecen sin definir a nivel global, quedando a discreción de cada país su elección. Lo anterior, determina 3 vías de formación que corren paralelas: una primera odontológica exclusiva, como en Chile y el resto de Latinoamérica; una segunda médica exclusiva, como en España, Italia y Francia; finalmente una tercera de DG, como en Alemania, Australia, Inglaterra, Holanda y otros países europeos y oceánicos, con una implementación parcial en Estados Unidos y Canadáa ${ }^{1-3}$.

Los resultados de este estudio están en línea con investigaciones previas $^{7,8}$ y editoriales ${ }^{9,10}$ del tema, mostrando una polarización de las opiniones respecto a la necesidad o validez del DG en CMF. Por una parte, existe favorable evidencia respecto a la integración de la formación médica (con obtención del título de médico-cirujano) en los programas de especialización en $\mathrm{CMF}^{1,7,9}$. En Estados Unidos y Canadá se han materializado programas integrados de 6 años de duración, a los que pueden ingresar dentistas o médicos, que al término obtienen: el segundo grado complementario, formación quirúrgica general y certificación en CMF, en tal sentido se logra una real integración médico-quirúrgicoodontológica y no sólo un complemento médico a la especialidad de CMF; mientras que en Reino Unido hay programas especiales para la obtención de la profesión faltante; en estos casos la obtención del segundo grado (médico-cirujano o cirujano-dentista) es en un tiempo menor, de 2 a 4 años, al de la vía tradicional que va de 4 a 6 años ${ }^{11}$.

Pero por otra parte, revisiones internacionales de la formación de especialistas en CMF reclaman la ausencia de diferencias entre cirujanos con y sin $\mathrm{DG}^{8,10}$, por lo que la DG se muestra como una opción poco eficiente, considerando que la duración y costos agregados no mejoran el resultado.

A pesar de la coherencia de los resultados de este trabajo con la literatura, algunas limitaciones de esta investigación deben tenerse en cuenta, tales como: la pequeña muestra de encuestados y un posible sesgo de autoselección o del voluntario hacia aquellos con una postura pro-DG.

Uno de los aspectos de mayor importancia reportado por los encuestados es la necesidad de contar con más competencias médicas, 3 de cada 5 cirujanos máxilofaciales declaran que en su práctica diaria las necesitarían, cuestión que parece de difícil resolución si no se accede a un programa de formación médica regular. Este resultado es algo contradictorio, pues la mayoría de los cirujanos también piensa que los programas de formación nacional tienen un buen nivel, esto podría explicarse por el hecho que muchos cirujanos son autoformados y por tanto sus necesidades no se condicen necesariamente con la formación entregada en los programas actuales.

Una proporción mayoritaria de cirujanos máxilofaciales chilenos apoyan la implementación del DG en el país, no necesariamente como la única vía de acceso a la especialidad, si no como una alternativa a la actual oferta de programas basados solo en el título odontológico. Sin embargo, de mayor interés es la relación existente entre una postura pro-DG con el ejercicio en ambiente hospitalario, el conocer la formación de DG o programas internacionales en esta modalidad, la necesidad de mayores conocimientos o habilidades médicas en la práctica diaria, la necesidad de contar con el DG para poder practicar el campo completo de la especialidad o la percepción que los programas de formación nacionales no se encuentran en un estándar internacional. Esta relación y las respuestas a la pregunta abierta refuerzan la existencia de una visión dicotómica acerca del DG, en la que la postura pro-DG se basa principalmente en el mejoramiento de las competencias del cirujano y las contrarias al DG en un mayor costo y duración (innecesario, injustificado e irrealizable en nuestro medio).

Dado lo anterior, posibles vías de solución para Chile son: la generación de programas integrados de formación en CMF para médicos o dentistas, como los existentes en Estados Unidos y Canadá ${ }^{1}$, que permitan una real integración médico-quirúrgicaodontológica con una duración y por ende costos aceptables; la creación de programas especiales de medicina para dentistas y de odontología para médicos, como los existentes en Reino Unido ${ }^{11}$; así como que las carreras odontológica y médica pueden acercar e igualar sus mallas curriculares de pregrado 
durante los primeros años de formación básica o pre-formación como es el sistema College, como ya sucede en países desarrollados ${ }^{11}$.

En virtud de estos resultados y el desarrollo económico del país, que recientemente ingresó a la OECD y ad portas de alcanzar un ingreso per cápita de país desarrollado, parece absolutamente posible y necesario iniciar una discusión propositiva acerca de la implementación de los programas de DG para formar a los futuros cirujanos máxilofaciales de Chile.

\section{Referencias}

1. Cartes-Velásquez R, Ramírez H. Dual-degree oral and maxillofacial surgery. Int J Odontostomat. 2011;5:1918.

2. UEMS, Section of Oro-Maxillo-Facial Surgery. Minuta 2008. The European Union of Medical Specialties. Bruselas, Bélgica 2008.

3. Assael LA. Training the future: Protecting the scope and diversity of oral and maxillofacial surgery. J Oral Maxillofac Surg. 2007;65:161-2.

4. Cartes-Velásquez R, Fernández M, Jara N, Ramírez H.
Dual-degree oral and maxillofacial surgery: evidence based view of chilean candidates. Int J Oral Maxillofac Surg. 2011:40:1153.

5. Assael L. Education of oral and maxillofacial surgeons in the 21st century. Int J Oral Maxillofac Surg. 2011;40:1012-3.

6. Gabrielli M. Training of oral and maxillofacial surgeons in South America. Int J Oral Maxillofac Surg. 2011;40:1012.

7. Herford AS, Pulsipher DA, Sinn DP. Integration of the medical degree in oral and maxillofacial surgery: A 10year follow-up. J Oral Maxillofac Surg. 2001;59:14716.

8. Laskin D. The past, present, and future of Oral and Maxillofacial Surgery. J Oral Maxillofac Surg. 2008;66:1037-40.

9. Assael L. The view from the third rail. J Oral Maxillofac Surg. 2010;68:713-4.

10. Sharafi A. Single degree and dual degree: we are all oral and maxillofacial surgeons. J Oral Maxillofac Surg. 2010;68:2926.

11. Cartes-Velásquez R, Moraga J. Ingreso de profesionales a la carrera de medicina: Revisión de la literatura. Rev Educ Cienc Salud 2013;10:59-63. 